\title{
Breast and Prostate Cancer Survivors in a Diabetic Cohort: Results from the Living With Diabetes Study
}

Adedayo A. Onitilo, MD, MSCR; Maria Donald, PhD; Rachel V. Stankowski, PhD; Jessica M. Engel, DNP, FNP-BC; Gail Williams, MSc, PhD and Suhail A. R. Doi, MD, PhD

\begin{abstract}
Objective: Diabetes is more common in cancer survivors than in the general population. The objective of the present study was to determine cancer frequency in a cohort of patients with diabetes and to examine demographic, clinical, and quality of life differences between cancer survivors and their cancer-free peers to inform better individualized care.
\end{abstract}

Methods: Self-reported survey data from 3,466 registrants with type 2 diabetes from Australia's National Diabetes Services Scheme (NDSS) were analyzed to compare relevant variables between cancer survivors and cancer-free patients. Analyses were focused on breast and prostate cancer to reflect the most common cancers in women and men, respectively.

Results: Five percent of diabetic women reported a history of breast cancer and $4.2 \%$ of men reported a history of prostate cancer. Diabetic patients with a history of breast or prostate cancer were older at time of survey and diabetes diagnosis, less likely to report metformin use (women), and more likely to have two or more comorbidities than their cancer-free peers. More diabetic prostate cancer survivors also reported problems with mobility and performing usual tasks. However, cancerfree diabetic subjects reported a lower diabetes-dependent quality of life than diabetic cancer survivors. There was no association between cancer survivorship and duration of diabetes, indices of glycemic control, obesity, or diabetic complications.

Conclusions: Cancer survivors comprise a significant minority of diabetic patients that are particularly vulnerable and may benefit from interventions to increase screening and treatment of other comorbidities and promote a healthy lifestyle.

Keywords: Diabetes, Patient characteristics; Metformin; Cancer; Cross-sectional survey

\begin{abstract}
A that the risk for several solid and hematologic malignancies is elevated in diabetic patients. ${ }^{1}$ Conversely, a higher frequency of diabetes is observed in cancer survivors than in the general population. ${ }^{2}$ The frequency of diagnosis is such that even minor reciprocal influences between cancer and diabetes may have a major impact on disease. ${ }^{1}$ The association between diabetes and cancer risk is complex and not welldefined, but is thought to occur as a consequence of
\end{abstract}

Corresponding Author: Adedayo A Onitilo, MD, MSCR, FACP, Marshfield Clinic Weston Center, 350I Cranberry Boulevard, Weston, WI 54476, Tel: 7I5-393-1400, Fax: 715-393-1399, Email: onitilo.adedayo@ marshfieldclinic.org

hyperglycemia and/or through underlying biologic factors that alter cancer risk, such as insulin resistance and hyperinsulinemia. ${ }^{3}$ Hyperglycemia and hyperinsulinemia may also be modulated by other risk factors common to cancer and diabetes, such as obesity or pharmacological agents for diabetes management. ${ }^{3}$ Similarly, the association between cancer survivorship and diabetes may be related to cancer treatment and/or unhealthy lifestyle choices. ${ }^{4,5}$

Received: February 13, 2013

Ist Revision: April 5, 2013

2nd Revision: May 7, 2013

Accepted: May 9, 2013
Funding: This study was supported by Queensland Health through the evaluation of the Queensland Strategy for Chronic Disease 2005-20I5. 
In a 2009 study using the United States Behavioral Risk Factor Surveillance System, the prevalence of diabetes in cancer survivors was $16.7 \%{ }^{6}$ In cancer survivors, incident or prevalent comorbid diabetes has been demonstrated to negatively impact overall and health-specific quality of life. ${ }^{7-10}$ While several studies have reported the prevalence of diabetes in cancer survivors with the goal of understanding and managing diabetes as a comorbidity in cancer survivors, the converse has not been examined. Understanding the rate of cancer survivorship in diabetic patients will help to understand the scope of the problem and size of the diabetic community for which cancer survivorship is also an issue. It is also important to understand how diabetic cancer survivors are different than diabetic patients in general in order to optimize care. The Living With Diabetes Study (LWDS) was undertaken to provide a comprehensive examination of temporal trends in satisfaction with care, quality of life, health care utilization, and disease progression in people living with diabetes in Queensland, Australia. ${ }^{11}$ The objective of this study was to use the state-wide LWDS database to report on the frequency of breast and prostate cancer survivors in this cohort of diabetic patients and to examine demographic, clinical, and quality of life differences in diabetic patients with and without a history of cancer. Because issues may be cancer-specific, we focused on the most common cancers in women and men, namely breast cancer in women and prostate cancer in men. We hypothesize that cancer survivors make up a small, but significant minority of diabetic patients and that these patients differ from their cancer-free peers with respect to demographics, clinical characteristics, and/or quality of life. Identifying such differences can help providers to focus on needs specific to this population.

\section{Methods}

\section{Study design and participants}

The Living with Diabetes Study (LWDS) is an ongoing longitudinal study of people with diabetes living in Queensland, Australia. Data from this study are contributing to a large scale evaluation of the state-wide Queensland Strategy for Chronic Disease 2005-2015 (QSCD) program whose goal is improvement of care relative to major chronic diseases, including diabetes. This paper reports on data collected at baseline in 2008 concerning breast and prostate cancer history among patients with diabetes.

The sampling frame was people with diabetes registered with Australia's National Diabetes Services Scheme (NDSS), an initiative of the Australian Government administered by Diabetes Australia. The primary purpose of the NDSS is to deliver diabetes-related products at subsidized prices to registrants, who must have a verified diagnosis for access. Eligibility criteria for study participants included registration with the NDSS, physical residence in Queensland with a valid postal address on record with the NDSS, age 18 years or older, diagnosis of type 1 or type 2 diabetes, and indication of interest in participation in research opportunities upon NDSS registration. Persons with gestational diabetes were excluded.
A sample of 14,439 registrants of the NDSS was invited to participate; each was mailed a questionnaire, information sheet, consent form, and reply-paid envelope. Completed questionnaires were returned by 3,951 participants, yielding a participation rate at baseline of $27 \%$. Ninety-five percent $(\mathrm{N}=3,761)$ of the participants had a diagnosis of type 2 diabetes. Because differences may be cancer-specific, we focused on breast and prostate cancer, and participants reporting a diagnosis of any other type of cancer were excluded from analysis resulting in a total of 3,466 eligible subjects. Type of diabetes diagnosis was verified using NDSS registration data. Individuals who took up the invitation to participate were largely similar to those who did not, with the exception that individuals were more likely to participate if they were aged over 60 years, and Indigenous Australians were less likely to participate. The final criterion, interest in receiving information about opportunities to participate in research, reduced the available population for sampling by about a third. Three policy target zones for the QSCD were over-sampled: an outer metropolitan area, a new urban development, and a coastal agricultural community. ${ }^{11}$

Ethics approval for the study was granted by the University of Queensland's Behavioural and Social Sciences Ethical Review Committee. Written informed consent was obtained from all study participants.

\section{Measures}

Of 347 primary variables in the 2008 baseline data file, the following demographic, clinical, and quality of life characteristics were extracted for this study: gender, age, age at diabetes diagnosis, self-reported HbAlc result, ever smoked, alcohol intake, diabetes-related complications, metformin use (duration of therapy was not recorded), co-morbid conditions, duration of diabetes, body mass index (BMI), quality of life measures, and history of breast or prostate cancer. Although age at cancer diagnosis was requested on the LWDS questionnaire, it was not reliably recorded or available for analysis. Therefore, all patients with a history of breast or prostate cancer were included, regardless of whether cancer was diagnosed before or after diabetes.

We used the EuroQoL group's EQ-5D ${ }^{12}$ to determine physical and mental health status and the Audit of Diabetes Dependent Quality of Life (ADDQoL) ${ }^{13}$ to determine diabetes-specific quality of life. Results of the former were scored in five dimensions and expressed in terms of any impairment (yes/ no) for each of the five dimensions, while the latter was expressed in terms of an impact score that can range from -9 to +3 indicating maximum negative to maximum positive impact on diabetes quality of life.

\section{Statistical procedures}

A cross-sectional analysis was performed on data from questionnaires completed in 2008. We compared groups with unpaired Student's $t$-tests and compared frequencies using $\chi^{2}$ tests. Comparisons were also adjusted for current age and 
Table 1: Baseline characteristics of adults with type 2 diabetes.

\begin{tabular}{|c|c|c|}
\hline & $\mathbf{N}^{\mathbf{a}}$ & $\begin{array}{c}\mathrm{n}(\%) \\
\text { or median (IQR) }\end{array}$ \\
\hline Cancer history & 3466 & $158(4.6)$ \\
\hline Breast cancer & 1547 & $77(5.0)$ \\
\hline Prostate cancer & 1919 & $81(4.2)$ \\
\hline Age (years) & 3466 & \\
\hline $18-44$ & & $217(6.3)$ \\
\hline $45-59$ & & $1094(31.5)$ \\
\hline $60-74$ & & 1731 (49.9) \\
\hline $75+$ & & $424(12.3)$ \\
\hline High co-morbidity (2+) & 3466 & 1675 (48.3) \\
\hline No. of complications & 3390 & $1(0-2)$ \\
\hline Duration of diabetes (years) & 3466 & $6(3-8)$ \\
\hline Male Gender & 3466 & $1919(55.4)$ \\
\hline Metformin use (yes) & 3466 & $1986(57.3)$ \\
\hline $\mathrm{HbA1c}$ & 2837 & \\
\hline$<6.5 \%$ & & $775(27.3)$ \\
\hline $6.5-7 \%$ & & $885(31.2)$ \\
\hline $7.1-8 \%$ & & $724(25.5)$ \\
\hline$>8 \%$ & & $453(16.0)$ \\
\hline HbA1c result score ${ }^{b}$ & 2837 & $2(1-3)$ \\
\hline Obesity (BMI > 30) & 3198 & $1674(52.3)$ \\
\hline Risky/high risk alcohol use & 3424 & $228(6.6)$ \\
\hline Have you ever smoked (yes) & 3088 & $1656(53.6)$ \\
\hline
\end{tabular}

BMI, body mass index; HbA1c, hemoglobin A1c

aNumber of respondents for whom data were available for each variable.

${ }^{b} 1 \leq 6.5 \% ; 2$ = 6.5-7\%; $3=7.1-8 \% ; 4 \geq 8 \%$ duration of diabetes to determine if differences were mediated through such non-modifiable factors. Multivariable logistic regression analysis was applied to model the association between duration of diabetes and cancer survivorship as prior research suggests that diabetes per se may be a risk factor for cancer. Statistically non-significant differences in confounders between duration groups may still cause an important confounding effect, and thus variables adjusted for in the latter analysis were selected based on either being known risk factors for cancer or related to glycemic control. Oversampling weights did not change the outcome of the final model when adjusted for, and thus were not used in model building. We used SPSS version 19 (IBM, Armonk, NY, USA) for all analyses.

\section{Results}

The general characteristics of the 3,466 subjects with type 2 diabetes are described in Table 1 . The majority of patients were in the 60 to 74-year-old age range, and the median duration of diabetes was 6 years. Pharmacological use surveyed among the subjects revealed that $57.3 \%$ were currently using metformin. Among the reference population, 158 subjects $(4.6 \%)$ had a history of breast or prostate cancer. There were 77 cases of breast cancer among 1,547 women $(5.0 \%)$, and 81 cases of prostate cancer among 1,919 men $(4.2 \%)$.

Compared to subjects with no history of cancer, women with a history of breast cancer and men with a history of prostate cancer were significantly older at time of survey, older at time of diabetes diagnosis (figure 1), less likely to report using metformin, and were more likely to have two or more comorbidities (tables 2 and 3). Breast cancer survivors were

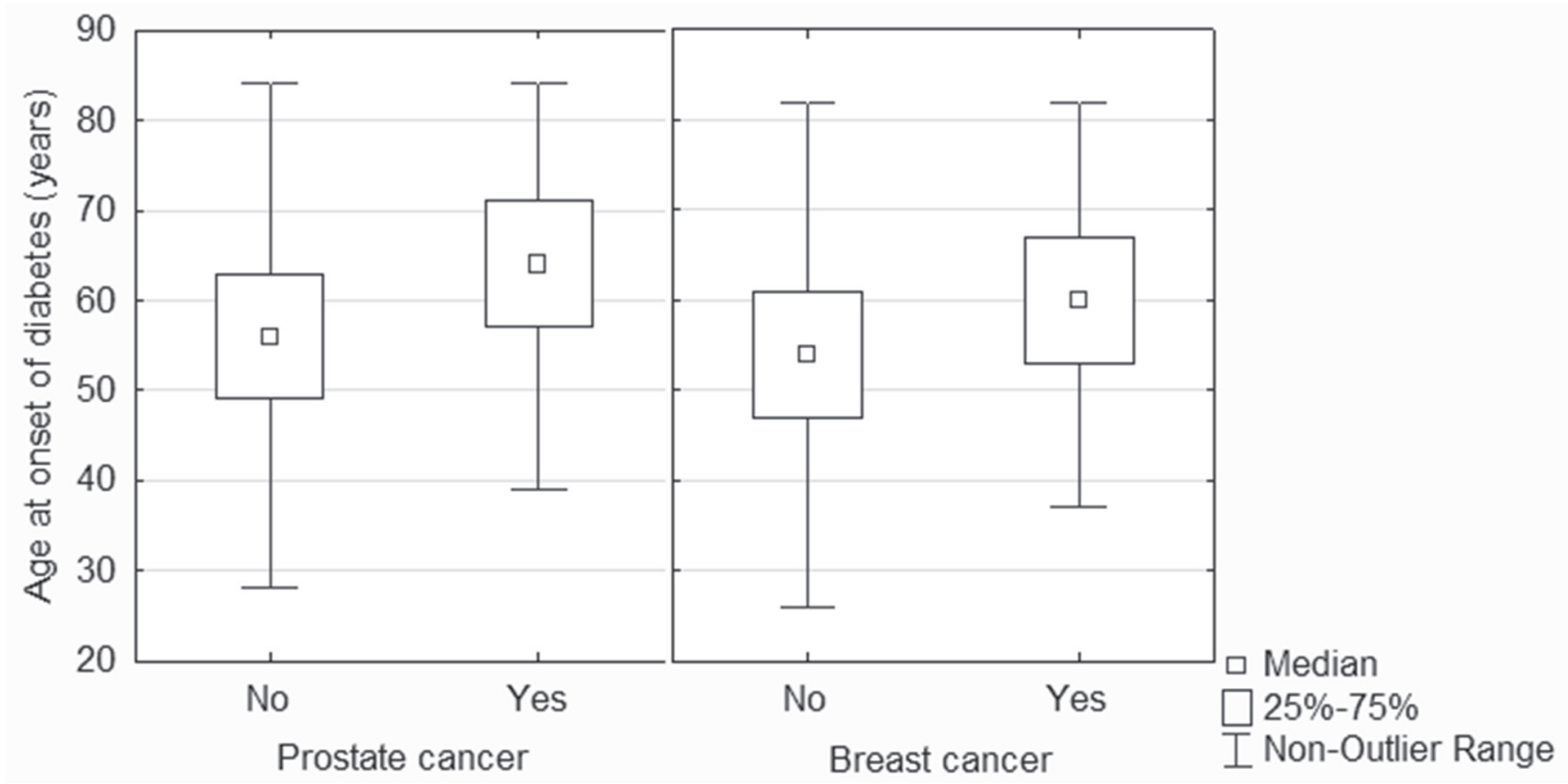

Figure 1. Box and whiskers plot comparing age at diabetes onset in diabetic patients with and without a history of prostate cancer (left) or breast cancer (right). 
Table 2: Comparisons between history of breast cancer and patient characteristics.

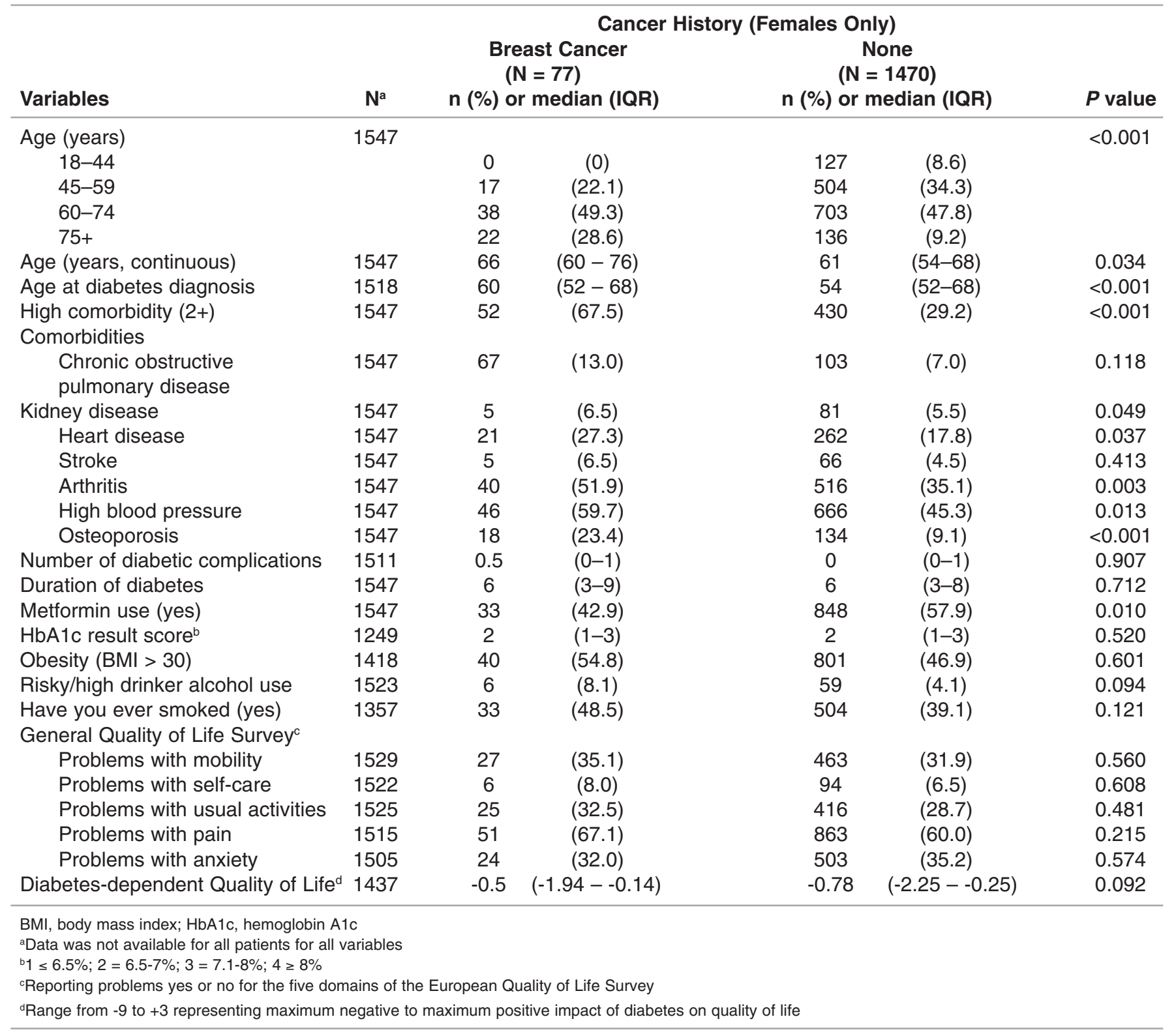

significantly more likely to report kidney disease, heart disease, arthritis, high blood pressure, and osteoporosis than their cancer-free diabetic peers (table 2). Prostate cancer survivors were significantly more likely to report heart disease and osteoporosis than their cancer-free diabetic peers, and there was a trend toward an increased rate of stroke as well (table 3). Few differences were observed in general quality of life measures; however, diabetic prostate cancer survivors were more likely to report problems with mobility and problems performing usual activities than diabetic patients with no history of cancer (table 3). There was a nonsignificant trend toward a lower diabetes-dependent quality of life score in patients with no history of cancer compared to either breast or prostate cancer survivors (tables 2 and 3). When all subjects were pooled, diabetes-dependent quality of life was significantly lower in diabetic subjects with no history of cancer $(-1.00 \mathrm{IQR}-2.41--0.32)$ compared to breast or prostate cancer survivors $(-0.71 \mathrm{IQR}-1.96--0.18$, $P=0.01)$.

The significant difference in age between subjects with and without a history of cancer raised the possibility that nonmodifiable factors like age and duration of diabetes may be key mechanisms influencing differences observed between the two groups. Additional analyses adjusted for age and duration of diabetes demonstrated that in women with a history of breast cancer, increased comorbidities, especially osteoporosis and arthritis, were independent of age (table 4). Similarly, in men with a history of prostate cancer increased comorbidities and problems with mobility were also independent of age (table 5).

Female subjects with a history of breast cancer and male subjects with a history of prostate cancer were divided into 
Table 3: Comparisons between history of prostate cancer and patient characteristics.

\begin{tabular}{|c|c|c|c|c|c|c|}
\hline \multirow[b]{2}{*}{ Variables } & \multicolumn{6}{|c|}{ Cancer History (Males Only) } \\
\hline & $\mathbf{N}^{\mathrm{a}}$ & \multicolumn{2}{|c|}{$\begin{array}{l}\text { Prostate Cancer }(\mathrm{N}=81) \\
\mathrm{n}(\%) \text { or median }(\mathrm{IQR})\end{array}$} & \multicolumn{2}{|c|}{$\begin{array}{l}\text { None }(\mathrm{N}=1838) \\
\mathrm{n}(\%) \text { or median (IQR) }\end{array}$} & \multirow{2}{*}{$\begin{array}{l}\boldsymbol{P} \text { value } \\
<0.001\end{array}$} \\
\hline & & & & & & \\
\hline $18-44$ & & 0 & $(0)$ & 90 & $(4.9)$ & \\
\hline $45-59$ & & 7 & $(8.6)$ & 566 & (30.8) & \\
\hline $60-74$ & & 39 & $(48.1)$ & 951 & $(51.7)$ & \\
\hline $75+$ & & 35 & (43.2) & 231 & $(12.6)$ & \\
\hline Age (years, continuous) & 1919 & 73 & $(66-79)$ & 63 & $(56-70)$ & $<0.001$ \\
\hline Age at diabetes diagnosis & 1883 & 64.5 & $(54-71.8)$ & 55 & $(48-63)$ & $<0.001$ \\
\hline High co-morbidity $(2+)$ & 1919 & 46 & $(56.8)$ & 407 & $(22.1)$ & $<0.001$ \\
\hline \multicolumn{7}{|l|}{ Comorbidities } \\
\hline $\begin{array}{l}\text { Chronic obstructive } \\
\text { pulmonary disease }\end{array}$ & 1919 & 7 & $(8.6)$ & 108 & $(5.9)$ & 0.305 \\
\hline Kidney disease & 1919 & 5 & $(6.2)$ & 97 & $(5.3)$ & 0.725 \\
\hline Heart disease & 1919 & 32 & (39.5) & 479 & $(26.1)$ & 0.007 \\
\hline Stroke & 1919 & 10 & (12.3) & 130 & $(7.1)$ & 0.074 \\
\hline Arthritis & 1919 & 27 & (333) & 463 & $(25.2)$ & 0.100 \\
\hline High blood pressure & 1919 & 39 & (48.1) & 770 & (41.9) & 0.265 \\
\hline Osteoporosis & 1919 & 6 & $(7.4)$ & 38 & $(2.1)$ & 0.002 \\
\hline Number of diabetic complications & 1879 & 1 & $(0-3)$ & 1 & $(0-2)$ & 0.145 \\
\hline Duration of diabetes & 1919 & 7 & $(4-9)$ & 6 & $(3-9)$ & 0.137 \\
\hline Metformin use (yes) & 1919 & 38 & $(46.9)$ & 1067 & $(58.0)$ & 0.047 \\
\hline $\mathrm{HbA1c}$ result score ${ }^{\mathrm{b}}$ & 1588 & 2 & $(1-3)$ & 2 & $(1-3)$ & 0.678 \\
\hline Obesity (BMI > 30) & 1780 & 31 & $(42.5)$ & 802 & $(47.0)$ & 0.449 \\
\hline Risky/high drinker alcohol use & 1901 & 5 & $(6.2)$ & 158 & $(8.7)$ & 0.448 \\
\hline Have you ever smoked (yes) & 1731 & 45 & $(60.0)$ & 1074 & $(64.8)$ & 0.390 \\
\hline \multicolumn{7}{|l|}{ General Quality of Life Surveyc } \\
\hline Problems with mobility & 1893 & 41 & $(51.2)$ & 526 & $(29.0)$ & $<0.001$ \\
\hline Problems with self-care & 1889 & 6 & $(7.5)$ & 135 & $(7.5)$ & 0.990 \\
\hline Problems with usual activities & 1898 & 28 & (35.0) & 447 & $(24.6)$ & 0.035 \\
\hline Problems with pain & 1886 & 45 & $(57.0)$ & 938 & (51.9) & 0.379 \\
\hline Problems with anxiety & 1879 & 22 & $(27.8)$ & 556 & (30.9) & 0.567 \\
\hline Diabetes-dependent Quality of Life & 1827 & -0.94 & $(-2.0--0.26)$ & -1.15 & $(-2.5--0.39)$ & 0.057 \\
\hline \multicolumn{7}{|c|}{$\begin{array}{l}\text { BMI, body mass index; HbA1c, hemoglobin A1c } \\
\text { aData was not available for all patients for all variables } \\
\text { b1 } \leq 6.5 \% ; 2=6.5-7 \% ; 3=7.1-8 \% ; 4 \geq 8 \%\end{array}$} \\
\hline \multicolumn{7}{|c|}{ 'Five domains of the European Quality of Life Survey } \\
\hline
\end{tabular}

tertiles based on duration of diabetes. Subjects with a diabetes duration of $\leq 4$ years were included in the low tertile, patients with a diabetes duration of 5 to 7 years were included in the middle tertile, and patients with a diabetes duration of $\geq 8$ years were included in the high tertile. The unadjusted odds ratio (OR) for breast or prostate cancer survivorship by duration of diabetes was not significant. The OR for breast cancer survivorship in the upper tertile was $1.03(95 \% \mathrm{CI}$ $0.64-1.68, P=0.90$ ) compared to the lower tertile. Similarly, the OR for prostate cancer survivorship in the upper tertile was $1.49(95 \%$ CI $0.95-2.34, P=0.08)$ suggesting no association. When potential confounding variables were adjusted for using a multivariable logistic regression model, there was still no association demonstrable between diabetes duration and breast or prostate cancer survivorship frequency. Variables adjusted for included age, BMI, metformin use, alcohol use, smoking, number of complications and glycemic control as determined by self-reported hemoglobin A1C levels.

\section{Discussion}

There is clear evidence of an association between diabetes and cancer, ${ }^{3}$ and data suggest that there is an increased frequency of diabetes in cancer survivors. ${ }^{2}$ Here, we sought to better understand the frequency of breast and prostate cancer survivors in the diabetic community and differences between diabetic patients with and without a history of cancer. In a defined cohort of individuals living with diabetes in Queensland, Australia, we found that the frequency of breast cancer survivorship among women was $5.0 \%$ and the frequency of prostate cancer survivorship among men was $4.2 \%$. Several demographic, clinical, and quality of life parameters were examined, and diabetic patients with a history of cancer were older at the time of survey and diabetes diagnosis, less likely to report metformin use, had more comorbidity, and had higher diabetes-dependent quality of life than their cancer-free peers, but were similar in terms of diabetes-specific indices compared to their cancer-free peers. Following adjustment for age and diabetes duration, 
significant differences in comorbidity remained, suggesting that the mechanism behind this difference was not simply age or diabetes duration related.

The findings of this study are consistent with the literature suggesting that the risk for breast and prostate cancer increases with age ${ }^{6}$ and that use of metformin reduces cancer risk. ${ }^{14}$ Similarly, several studies have reported that cancer survivors with diabetes have more comorbidities than cancer survivors without diabetes. ${ }^{2}$ It is interesting that the same holds true for diabetic cancer survivors compared to diabetic subjects with no history of cancer and may be related to the late-term effects of cancer and/or cancer treatment. ${ }^{15}$ In our study, vascular risks were increased in cancer patients as evidenced by increased rates of kidney disease, heart disease, and high blood pressure in breast cancer survivors and increased heart disease and a trend toward increased stroke in prostate cancer survivors. Vascular risks were also increased in diabetes, suggesting that surveillance and early intervention may be especially important for diabetic cancer survivors. Cancer therapy, especially chemotherapy and endocrine therapy, is known to contribute to osteoporosis ${ }^{16}$ and to cardiovascular risk. ${ }^{17,18}$ While some of these problems may be related to cancer survivors being older, it does not detract from the fact that they need to be addressed when dealing with such patients, regardless of whether it is a direct effect of cancer or not.

The finding that breast and prostate cancer survivors were generally diagnosed with diabetes at an older age is particularly interesting given the literature suggesting that diabetes per se may be a risk factor for cancer. ${ }^{19}$ We therefore took particular interest in determining if duration of diabetes was independently associated with cancer survivorship and found that this was not the case. This latter finding suggests that diabetes-related factors of control and complications may not be key factors influencing cancer survivorship but contribute simply to the burden of comorbidities. This is consistent with our observation that traditional risk factors for both diabetes and cancer, including obesity and glycemic control, were not significantly different in diabetic subjects who were or were not breast or prostate cancer survivors. Similarly, there was no difference in the rate of diabetes complications in either group. This suggests that management of diabetes is similar regardless of cancer history, and that worsening measures of glycemic control are more related to increased diabetes duration than to whether or not patients have a history of cancer.

It has been suggested that some cancer survivors may be so focused on recurrence that other aspects of their health may be neglected, including lifestyle changes that may contribute to the development of diabetes and management of existing diabetes. ${ }^{10}$ Among cancer survivors, those with comorbid conditions, including diabetes, report a higher rate of disability and overall poorer health than those without comorbidities. ${ }^{10}$ In general, cancer survivors with diabetes are more likely to report that cancer has affected their overall health and are more likely to suffer from several other comorbid conditions including arthritis, cataracts, circulation problems, dizziness, strokes, hearing impairment, and heart, kidney, liver, and thyroid problems than cancer survivors without diabetes. ${ }^{2}$ For

Table 4: Age and diabetes-duration adjusted logistic regression for breast cancer.

\begin{tabular}{|c|c|c|c|}
\hline Variables & Odds Ratio (OR) & $95 \% \mathrm{Cl}$ & $P$ value \\
\hline Age at diabetes diagnosis & 0.988 & $0.960-1.017$ & 0.407 \\
\hline High co-morbidity $(2+)$ & 0.222 & $0.135-0.365$ & $<0.001$ \\
\hline \multicolumn{4}{|l|}{ Comorbidities } \\
\hline Chronic obstructive pulmonary disease & 0.537 & $0.265-1.089$ & 0.085 \\
\hline Kidney disease & 0.908 & $0.350-2.356$ & 0.843 \\
\hline Heart disease & 0.789 & $0.459-1.357$ & 0.392 \\
\hline Stroke & 0.876 & $0.335-2.290$ & 0.787 \\
\hline Arthritis & 0.622 & $0.389-0.944$ & 0.047 \\
\hline High blood pressure & 0.621 & $0.388-0.996$ & 0.048 \\
\hline Osteoporosis & 0.476 & $0.265-0.853$ & 0.013 \\
\hline Number of diabetic complications & 1.055 & $0.864-1.290$ & 0.599 \\
\hline Metformin use (yes) & 0.625 & $0.390-1.002$ & 0.051 \\
\hline Obesity (BMI > 30) & 0.863 & $0.523-1.424$ & 0.564 \\
\hline Risky/high drinker alcohol use & 0.479 & $0.198-1.161$ & 0.103 \\
\hline Have you ever smoked (yes) & 1.576 & $0.960-2.586$ & 0.072 \\
\hline \multicolumn{4}{|l|}{ General Quality of Life Survey ${ }^{a}$} \\
\hline Problems with mobility & 1.050 & $0.639-1.725$ & 0.847 \\
\hline Problems with self-care & 0.857 & $0.357-2.052$ & 0.728 \\
\hline Problems with usual activities & 0.951 & $0.576-1.571$ & 0.845 \\
\hline Problems with pain & 0.842 & $0.511-1.391$ & 0.504 \\
\hline Problems with anxiety & 1.004 & $0.606-1.664$ & 0.987 \\
\hline Diabetes-dependent Quality of Life & 1.062 & $0.911-1.239$ & 0.440 \\
\hline
\end{tabular}

$\mathrm{BMI}$, body mass index; $\mathrm{Cl}$, confidence interval

aFive domains of the European Quality of Life Survey 
Table 5: Age and diabetes-duration adjusted logistic regression for prostate cancer.

\begin{tabular}{|c|c|c|c|}
\hline Variables & Odds Ratio (OR) & $95 \% \mathrm{Cl}$ & $P$ value \\
\hline Age at diabetes diagnosis & 0.994 & $0.968-1.020$ & 0.637 \\
\hline High co-morbidity $(2+)$ & 0.268 & $0.168-0.427$ & $<0.001$ \\
\hline \multicolumn{4}{|l|}{ Comorbidities } \\
\hline Chronic obstructive pulmonary disease & 0.753 & $0.331-1.711$ & 0.498 \\
\hline Kidney disease & 1.161 & $0.442-3.053$ & 0.762 \\
\hline Heart disease & 0.856 & $0.531-1.378$ & 0.521 \\
\hline Stroke & 0.889 & $0.436-1.814$ & 0.747 \\
\hline Arthritis & 0.943 & $0.577-1.539$ & 0.814 \\
\hline High blood pressure & 0.793 & $0.502-1.253$ & 0.320 \\
\hline Osteoporosis & 0.436 & $0.173-1.096$ & 0.077 \\
\hline Number of diabetic complications & 0.942 & $0.790-1.123$ & 0.505 \\
\hline Metformin use (yes) & 0.908 & $0.569-1.451$ & 0.688 \\
\hline Obesity (BMI > 30) & 0.679 & $0.408-1.129$ & 0.136 \\
\hline Risky/high drinker alcohol use & 1.157 & $0.454-2.947$ & 0.761 \\
\hline Have you ever smoked (yes) & 0.684 & $0.420-1.113$ & 0.126 \\
\hline \multicolumn{4}{|l|}{ General Quality of Life Surveya } \\
\hline Problems with mobility & 0.567 & $0.353-0.909$ & 0.018 \\
\hline Problems with self-care & 1.205 & $0.497-2.919$ & 0.680 \\
\hline Problems with usual activities & 0.750 & $0.459-1.223$ & 0.249 \\
\hline Problems with pain & 0.912 & $0.571-1.456$ & 0.699 \\
\hline Problems with anxiety & 0.914 & $0.544-1.534$ & 0.733 \\
\hline Diabetes-dependent Quality of Life & 1.145 & $0.965-1.358$ & 0.121 \\
\hline
\end{tabular}

example, using a health utility score as a measure of quality of life, $\mathrm{Ko}_{\mathrm{ot}} \mathrm{al}^{7}$ reported that health utility scores increased over time following acute illness with breast cancer. However, diabetes was predictive of a lower health utility score. ${ }^{7}$ Similarly, Thong et $\mathrm{al}^{9}$ reported that men with diabetes diagnosed either before or after prostate cancer diagnosis have poorer general health and lower health-related quality of life than prostate cancer survivors without diabetes. In the present study, diabetic prostate cancer survivors were more likely to report problems with mobility and performing usual activities than diabetic men with no history of cancer. We did not, however, observe any difference in general quality of life between diabetic breast cancer survivors and diabetic women with no history of cancer. Interestingly, diabetic subjects with no history of cancer had a lower diabetes-dependent quality of life score than diabetic cancer survivors, suggesting that cancer survivors perceive diabetes to have a lesser impact on their quality of life. It is possible that this reflects a difference in perspective related to cancer survival, whereby diabetes is overshadowed by a decrease in quality of life as a result of cancer.

Cancer survivors appear to hold a unique position as a significant minority of the overall diabetic population. Evidence suggests that individuals with diabetes are at a higher risk for cancer than those without diabetes. ${ }^{20}$ It follows that cancer survivors with diabetes may have a greater risk for cancer recurrence than cancer survivors without diabetes. Unfortunately, diabetic patients are less likely to undergo regular cancer screening, including mammography, than their non-diabetic counterparts. ${ }^{21,22}$ Additionally, findings from the present study suggest that diabetic subjects with a history of breast or prostate cancer have a greater number of comorbidities than those with no history of cancer, and data from the Surveillance Epidemiology End Results (SEER) database strongly suggests that cancer survivors die of noncancer causes at a higher rate than the general population. ${ }^{4}$ Therefore, diabetic cancer survivors may be a particularly vulnerable population and may benefit greatly from health and lifestyle interventions, such as promotion of a healthful diet and active, non-smoking lifestyle, as described by Stull et $\mathrm{al},{ }^{4}$ and targeted cancer screening.

One important limitation of the present study is an unknown date of cancer diagnosis. It is impossible to know if subjects had prevalent diabetes at the time of cancer diagnosis or incident diabetes that was diagnosed after the cancer diagnosis. Even if cancer diagnosis date were known, determining whether cancer occurred before or after diabetes onset is still imprecise as hyperinsulinemia and progressive hyperglycemia are also present in the pre-diabetes phase. However, in a study by Thong et al, ${ }^{9}$ diabetes was found to negatively impact on health-related quality of life in prostate cancer survivors whether diabetes onset occurred before or after prostate cancer diagnosis, although incident diabetes had a lesser effect. Additional study limitations include crosssectional design, data collection by self-report, survivorship bias, and healthy subject bias. The cross-sectional study design makes the directionality of any associations difficult to establish. The fact that we did not demonstrate a link between increasing duration of diabetes and cancer survivorship is complicated by the long preclinical prediabetic state with a 
similar metabolic and physiologic internal environment as in an overt diabetic state. Thus, exact duration of exposure is difficult to ascertain. The effect and duration of diabetic treatments, such as metformin, further complicates matters. Findings are also limited by the fact that medication regimens and co-morbid diagnoses were self-reported and, therefore, reliant on recall. Self-report in general presents difficulties. Hewitt et $\mathrm{a}^{23}$ found that self-report tends to underestimate the prevalence of cancer compared to use of a tumor registry by approximately $14 \%$ for males with prostate cancer and $20 \%$ for women with breast cancer. Furthermore, the response rate for subjects consenting to participate in research was low, yet consistent with research showing that participation rates in large cohort studies appear to be declining from about $80 \%$ to $30 \%$ or $40 \%$ over the past several decades. ${ }^{24}$ It is possible that prevalence estimates of the collected variables underestimated cancer rates in the entire LWDS population. However, analysis of self-report response patterns in the Prostate Cancer Outcomes Study suggest that with respect to comorbid conditions, including diabetes, response consistencies as high as $92 \%$ were obtained, and consistency tended to be better in patients taking prescription medication for their condition. ${ }^{25}$ Finally, cancer and its treatment is often accompanied by significant morbidities that may affect individual interest in survey participation, thus creating a healthy subject bias, which may act toward decreasing cancer prevalence rate. Similarly, this study is limited to a comparison of cancer survivors, rather than those with cancer diagnoses, because mortality of subjects before study commencement as a result of cancer may result in a survivorship bias. This bias makes comparison of cancer versus no cancer difficult since healthier subjects who have survived longer are more likely to be included.

\section{Implications}

Cancer survivors have an older age at diabetes onset and more comorbidity. Specifically, vascular risks are increased in diabetes and cancer, and this population may require increased surveillance and early intervention. There was less metformin use seen in women, and given that metformin decreases insulin resistance and is associated with a reduced risk of cancer, its use should be encouraged in the elderly diabetic populations. Better diabetes-dependent QoL was seen in women, and this could be because diabetes may be overshadowed by a cancer-related decrease in quality of life. Finally, a similar diabetes duration, glycemic control, body mass index, and number of complications suggest that diabetes-specific indices are comparable; therefore, older age at onset and increase comorbidity are independent of glycemic control or obesity. Finally, breast or prostate cancer survivorship is a concern for as many as 5\% to $10 \%$ of people living with diabetes, and therefore preventive and risk reduction measures, as well as early diagnosis and treatment of other medical conditions such as cardiovascular disease or osteoporosis in cancer survivors with diabetes should be encouraged. We conclude that cancer survivors comprise a significant minority of diabetic patients that are particularly vulnerable and may benefit from health interventions to increase screening and treatment of other comorbidities and promote a healthy lifestyle.

\section{Acknowledgements}

The authors thank Marie Fleisner of the Marshfield Clinic Research Foundation's Office of Scientific Writing and Publication for editorial assistance in the preparation of this manuscript.

\section{References}

1. Vigneri P, Frasca F, Sciacca L, Pandini G, Vigneri R. Diabetes and cancer. Endocr Relat Cancer 2009;16:1103-1123.

2. Stava CJ, Beck ML, Feng L, Lopez A, Busaidy N, Vassilopoulou-Sellin R. Diabetes mellitus among cancer survivors. J Cancer Surviv 2007;1:108-115.

3. Giovannucci E, Harlan DM, Archer MC, Bergenstal RM, Gapsture SM, Habel LA, Pollak M, Regensteiner JG, Yee D. Diabetes and cancer: a consensus report. Diabetes Care 2010;33:1674-1685.

4. Stull VB, Snyder DC, Denmark-Wahnefried W. Lifetsyle interventions in cancer survivors: designing programs that meet the needs of this vulnerable and growing population. $\mathrm{J}$ Nutr 2007;137:243S-248S.

5. von Gruenigen VE, Waggoner SE, Frasure HE, Kavanagh MB, Janata JW, Rose PG, Courneya KS, Lerner E. Lifestyle challenges in endometrial cancer survivorship. Obstet Gynecol 2011;117:93-100.

6. Underwood JM, Townsend JS, Stewart SL, Buchannan N, Ekwueme DU, Hawkins NA, Li J, Peaker B, Pollack LA, Richards TB, Rim SH, Rohan EA, Sabatino SA, Smith JL, Tai E, Townsend GA, White A, Fairley TL; Division of Cancer Prevention and Control, National Center for Chronic Disease Prevention and Health Promotion, Centers for Disease Control and Prevention (CDC). Surveillance of demographic characteristics and health behaviors among adult cancer survivors-Behavioral Risk Factor Surveillance System, United States, 2009. MMWR Surveill Summ 2012;61:1-23.

7. Ko CY, Maggard M, Livingston EH. Evaluation health utility in patients with melanoma, breast cancer, colon cancer, and lung cancer: a nationwide, population-based assessment. J Surg Res 2003;114:1-5.

8. Mols F, Aquarius AE, Essink-Bot ML, Aaronson NK, Kil PJ, van de Poll-Franse LV. Does diabetes mellitus as a comorbid condition affect the health-related quality of life in prostate cancer survivors? Results of a population-based observational study. BJU Int 2008;102:1594-1600.

9. Thong MS, van de Poll-Franse L, Hoffman RM, Albertsen PC, Hamilton AS, Stanford JL, Penson DF. Diabetes mellitus and health-related quality of life in prostate cancer: 5-year results from the Prostate Cancer Outcomes Study. BJU Int 2011;107:1223-1231.

10. Edgington A, Morgan MA. Looking beyond recurrence: comorbidities in cancer survivors. Clin J Oncol Nurs 2011;15:E3-E12.

11. Donald M, Dower J, Ware R, Mukandi B, Parekh S, Bain C. Living with diabetes: Rationale, study design and baseline characteristics for an Australian prospective cohort study. BMC Public Health 2012;12:8.

12. Brooks R. EuroQoL: the current state of play. Health Policy 1996;37:53-72.

13. Bradley C, Todd C, Gorton T, Symonds E, Martin A, Plowright $\mathrm{R}$. The development of an individualized questionnaire measure of perceived impact on diabetes quality of life: the ADDQoL. Qual Life Res 1999;8:79-91.

14. Taubes G. Cancer research. Cancer prevention with a diabetes pill? Science 2012;335:29. 
15. Khan NF, Mant D, Carpenter L, Forman D, Rose PW. Longterm health outcomes in a British cohort of breast, colorectal and prostate cancer survivors: a database study. Br J Cancer 2011;105:S29-S37.

16. Pfeilschifter J, Diel IJ. Osteoporosis due to cancer treatment: Pathogenesis and management. J Clin Oncol 2000:1570-1593.

17. Yeh ET, Tong AT, Lenihan DJ, Yusuf SW, Swafford J, Champion C, Durand JB, Gibbs H, Zafarmand AA, Ewer MS. Cardiovascular complications of cancer therapy: diagnosis, pathogenesis, and management. Circulation 2004;109:3122-131.

18. Nguyen PL, Je Y, Schutz FA, Hoffman KE, Hu JC, Parekh A, Beckman JA, Choueiri TK. Association of androgen deprivation therapy with cardiovascular death in patients with prostate cancer: a meta-analysis of randomized trials. JAMA 2011;306:2359-2366.

19. Onitilo AA, Engel JM, Glurich I, Stankowski RV, Williams GM, Doi SA. Diabetes and cancer II: role of diabetes medications and influence of shared risk factors. Cancer Causes Control 2012;23:991-1008.

20. Chowdhury TA. Diabetes and cancer. QJM. 2010;103:905-915.

21. Lipscombe LL, Hux JE, Booth GL. Reduced screening mammography among women with diabetes. Arch Intern Med 2005;165:2090-2095.

22. Jimenez-Garcia R, Hernandez-Barrera V, Carrasco-Garrido P, Gil A. Prevalence and predictors of breast and cervical cancer screening among Spanish women with diabetes. Diabetes Care 2009;32:1470-1472.

23. Hewitt M, Breen N, Devesa S. Cancer prevalence and survivorship issues: analyses of the 1992 National Health Interview Survey. J Natl Cancer Inst 1999;91:1480-1486.

24. Nohr EA, Frydenberg M, Henriksen TB, Olsen J. Does low participation in cohort studies induce bias? Epidemiology 2006;17:413-418.

25. Klabunde CN, Reeve BB, Harlan LC, Davis WW, Potosky AL. Do patients consistently report comorbid conditions over time?: results from the prostate cancer outcomes study. Med Care 2005;43:391-400.

\section{Author Affiliations}

Adedayo A. Onitilo, MD, MSCR ${ }^{*,}$; Maria Donald, PhD*;

Rachel V. Stankowski, PhD; Jessica M. Engel, DNP, FNP-BC $C^{\xi}$; Gail Williams, MSc, PhD* and Suhail A. R. Doi, $M D, P h D^{*}$

"School of Population Health, University of Queensland, Brisbane, Queensland, Australia

'Department of Hematology/Oncology, Marshfield Clinic, Weston Center, Weston, Wisconsin, USA

*Marshfield Clinic Research Foundation, Marshfield,

Wisconsin, USA

${ }^{3}$ Marshfield Clinic Cancer Care at St. Michael's, Stevens Point, Wisconsin USA 